\title{
An Evidence-Based Review of Quantitative SPECT Imaging and Potential Clinical Applications
}

\author{
Dale L. Bailey ${ }^{1,2}$ and Kathy P. Willowson ${ }^{3}$ \\ ${ }^{1}$ Department of Nuclear Medicine, Royal North Shore Hospital, St. Leonards, Australia; ${ }^{2}$ Discipline of Medical Radiation Sciences, \\ University of Sydney, Sydney, Australia; and ${ }^{3}$ School of Physics, University of Sydney, Sydney, Australia
}

Learning Objectives: On successful completion of this activity, participants should be able to (1) review the current status of SPECT imaging, with an emphasis on clinical applications for quantitative interpretations; (2) consider the requirements for quantitative SPECT imaging-instrumentation, software, and image calibration; and (3) acquire knowledge of the capabilities of quantitative SPECT with a view to developing new clinical applications.

Financial Disclosure: Dr. Bailey is an employee of NSW Health, University of Sydney, and an investigator for NHMRC, ARC, Cure Cancer Australia. Dr. Willowson is an employee of the University of Sydney and is supported by the Australian Research Council and the NSW Cancer Council in conjunction with the Cure Cancer Australia Foundation. The authors of this article have indicated no other relevant relationships that could be perceived as a real or apparent conflict of interest.

CME Credit: SNMMI is accredited by the Accreditation Council for Continuing Medical Education (ACCME) to sponsor continuing education for physicians. SNMMI designates each JNM continuing education article for a maximum of 2.0 AMA PRA Category 1 Credits. Physicians should claim only credit commensurate with the extent of their participation in the activity. For CE credit, participants can access this activity through the SNMMI Web site (http:// www.snmmi.org/ce_online) through January 2016.

SPECT has traditionally been regarded as nonquantitative. Advances in multimodality $\gamma$-cameras (SPECT/CT), algorithms for image reconstruction, and sophisticated compensation techniques to correct for photon attenuation and scattering have, however, now made quantitative SPECT viable in a manner similar to quantitative PET (i.e., $\mathrm{kBq} \cdot \mathrm{cm}^{-3}$, standardized uptake value). This review examines the evidence for quantitative SPECT and demonstrates clinical studies in which the accuracy of the reconstructed SPECT data has been assessed in vivo. SPECT reconstructions using CT-based compensation corrections readily achieve accuracy for ${ }^{99 m}$ Tc to within $\pm 10 \%$ of the known concentration of the radiotracer in vivo. Quantification with other radionuclides is also being introduced. SPECT continues to suffer from poorer photon detection efficiency (sensitivity) and spatial resolution than PET; however, it has the benefit in some situations of longer radionuclide half-lives, which may better suit the biologic process under examination, as well as the ability to perform multitracer studies using pulse height spectroscopy to separate different radiolabels.

Key Words: SPECT (single-photon emission computed tomography); quantification; scatter correction; attenuation correction; validation

J Nucl Med 2013; 54:83-89

DOI: 10.2967/jnumed.112.111476

Q uantitative emission tomography is a powerful investigative tool in clinical practice and biomedical research. The two principal forms of emission tomography using radionu-

Received Sep. 11, 2012; revision accepted Dec. 7, 2012.

For correspondence or reprints contact: Dale L. Bailey, Department of Nuclear Medicine, Royal North Shore Hospital, St. Leonards, Australia 2065. E-mail: dale.bailey@sydney.edu.au

COPYRIGHT (c) 2013 by the Society of Nuclear Medicine and Molecular Imaging, Inc. clide-labeled compounds are SPECT and PET. Although PET has a tremendous sensitivity advantage and higher spatial resolution over conventional SPECT, mostly because of the methods of collimation used in each, SPECT arguably has some advantages over PET. The physical half-lives for many SPECT radionuclides are generally longer and more aligned with the biologic half-lives of physiologic processes of interest; radiotracers are readily available and do not require relatively close proximity to a medical cyclotron and a rapid distribution network; there is the potential for simultaneous multitracer studies with different radionuclides examining different biologic pathways in a single imaging session; and the systems are of lower cost and have a much greater installed base worldwide. As the $\gamma$-camera/SPECT component in a SPECT-only system is the same as that in a combined SPECT/CT system, we will use the terms SPECT and SPECT/CT interchangeably. In doing so, we emphasize that the SPECT acquisition and performance are independent of whether the system has a CT scanner attached.

Since the inception of PET, PET systems have been designed to produce reconstructed images that are inherently quantitative (i.e., the reconstructed data are in units of radioactivity per unit volume $\left[\mathrm{kBq} \cdot \mathrm{cm}^{-3}\right]$ ) because the correction for photon absorption is relatively straightforward to apply and in early 2-dimensional scanners the amount of scattered radiation detected was low enough $(<5 \%)$ to be ignored. In SPECT, however, these corrections have been more challenging, and to this day much of the SPECT imaging performed is without correction for photon absorption (attenuation) or scattered radiation. Put simply, the dogma has arisen that PET is quantitative and SPECT is not, as is demonstrated by the following recent example from this journal (1): “...PET is superior to SPECT in both sensitivity and spatial resolution. Furthermore, PET enables quantitation of tissue radioactivity concentrations." 
Today, however, 3-dimensional PET systems record a large fraction of scattered events (from 35\% to $>50 \%$ of all detected coincident events) such that the scatter correction required is on the same order as, or even greater than, that required in SPECT. Although various strategies have been proposed to produce quantitative SPECT data, such as combined use of transmission radionuclide sources (e.g., ${ }^{153} \mathrm{Gd}$ or ${ }^{241} \mathrm{Am}$ ) with simultaneous emission imaging to directly measure emission photon absorption $(2,3)$, it has been the relatively recent introduction of combined SPECT/ CT scanners that has given renewed impetus to this goal. The CT data complement the SPECT data by providing information about the density of the body tissues, which can be used in algorithms that aim to correct for photons that have been Compton-scattered or attenuated within the body. Although the data from CT are not absolutely essential for producing quantitative SPECT images, the ready availability of coregistered datasets of SPECT and CT has certainly assisted in the ability to apply corrections for scattered and attenuated photons more easily.

In this review, we will concentrate on the latest developments to achieve quantitative SPECT reconstruction based on the conventional $\mathrm{NaI}(\mathrm{Tl}) \gamma$-camera, whether used with or without a combined CT system. We do not consider the recently introduced solid-state (cadmium zinc telluride) SPECT systems in this discussion.

\section{QUANTIFICATION IN EMISSION TOMOGRAPHY}

The requirements for producing quantitative data in emission tomography are the same for PET as for SPECT. The main features are a reconstruction algorithm that behaves in a linear fashion in terms of the reconstructed radioactivity concentration, an algorithm to compensate for photon absorption within the body, an algorithm to remove scattered radiation from the data, and the ability to calibrate the reconstructed data in $\mathrm{kBq} \cdot \mathrm{cm}^{-3}$. There are several other factors that may influence the quantitative accuracy of reconstructed PET or SPECT data, including a decreased apparent radioactivity concentration in objects less than approximately 3 times the spatial resolution of the system and therefore affected by the partial-volume effect, counting rate losses due to dead time within the instrumentation, radioactive decay during the acquisition process, and corrections and normalizations for spatial and temporal variations in detector response. A subtle final point is that PET reconstruction has traditionally been performed with voxel values in units of radioactivity per unit volume $\left(\mathrm{kBq} \cdot \mathrm{cm}^{-3}\right)$, that is, an image of radioactivity concentration, whereas SPECT has tended to be reconstructed as a count-based image. The difference is accentuated when a zoom is applied during reconstruction: the image reconstructed as a radioactivity concentration with or without a software zoom will represent those data with a constant numeric value per voxel; that is, the concentration of radioactivity is independent of the zoom applied. Conversely, the count-conserving algorithm in SPECT will keep the total number of recon- structed events constant and, as the object reconstructed with increasing zoom appears larger because of the magnification applied in software, the value contained in each voxel will be decreased as the total number of counts is held constant. This difference can probably be traced back to the "PET is quantitative, SPECT is not" dogma. Conversion between these two situations is, of course, relatively straightforward, even after reconstruction.

The reconstruction algorithm of choice today in emission tomography is based on one of the statistical iterative methods, as opposed to the classic analytic reconstruction methodology of filtered backprojection, with many applications using the block-iterative, ordered-subset maximum-likelihood expectation maximization algorithm (OSEM) (4). This approach has numerous attractive features, including the ability to model physical characteristics of the acquisition process in the reconstruction to enhance image quality and accuracy, and the ability to better control the signal-to-noise characteristics of the final image. Most reconstruction software today includes optional scatter and attenuation correction and, increasingly, depthdependent resolution recovery (sometimes referred to as pointspread function correction or, alternatively, high definition).

Once the quantitative accuracy of the SPECT image reconstruction in $\mathrm{kBq} \cdot \mathrm{cm}^{-3}$ has been established, it is then possible to review the data by applying parameters such as a standardized uptake value (SUV) using knowledge of the administered amount of radioactivity, delay from injection until scanning for radioactive decay correction, and patient weight/volume. An example comparing the quantitative accuracy of PET and SPECT images expressed as an SUV is shown in Figure 1.

The data in Figure 1 were acquired using the novel in-line SPECT/CT system developed in our department and consisting of a dual-head $\gamma$-camera (SKYLight) combined with a single-slice helical CT scanner (PQ5000) (5) and a stateof-the-art lutetium oxyorthosilicate-based PET/CT scanner with time-of-flight capability and an extended axial field of view (Biograph mCT/64) (6). The test object used was the International Electrotechnical Commission (IEC) body phantom with spheric inserts as described in the latest National Electrical Manufacturers Association PET evaluation test procedures (7) except that in this case the largest 2 spheres, instead of containing nonradioactive water, were filled with the same radioconcentration of activity as were the other spheres. The ratio for the radioconcentration was 7.3:1 for PET and 8.2:1 for SPECT. Scans were acquired under typical clinical imaging conditions. The SPECT data were reconstructed using an OSEM algorithm (4) with attenuation and scatter correction based on the CT data. No partial-volumeeffect correction or point-spread-function resolution recovery was applied to the SPECT data. Further details of the processing and system calibration can be found in an article by Willowson et al. (8). The PET data were reconstructed using 3-dimensional OSEM, including time-offlight weighting and point-spread-function resolution recovery. The amount of radioactivity introduced into the phantom 


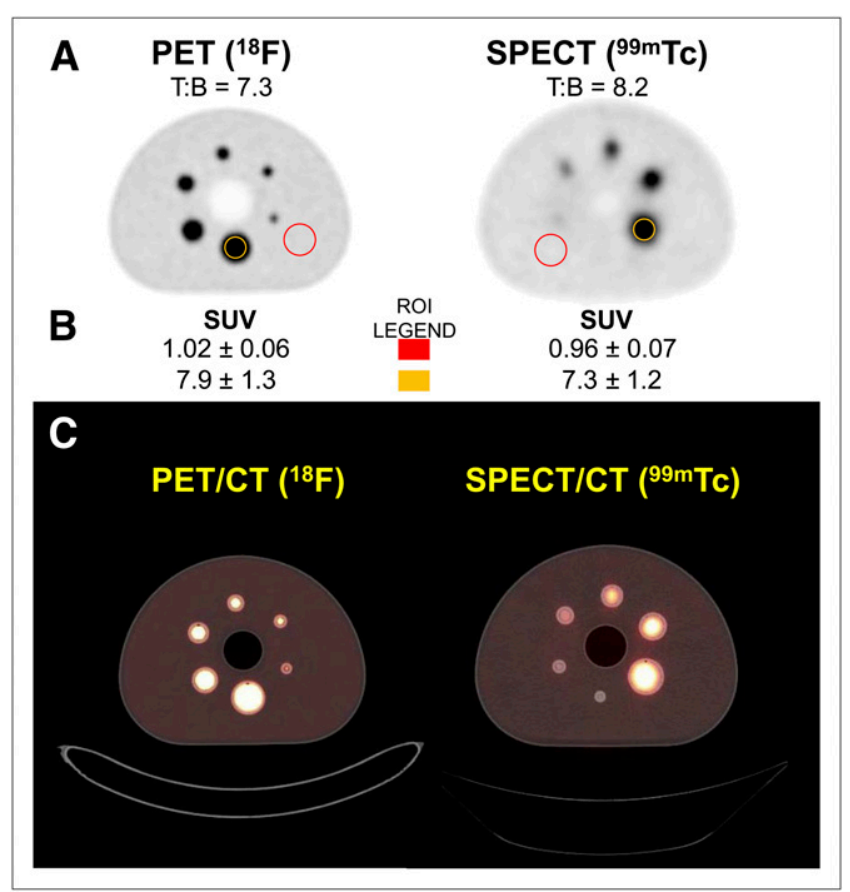

FIGURE 1. Comparison of quantitative PET $\left({ }^{18} \mathrm{~F}\right)$ and SPECT $\left({ }^{99 \mathrm{~m} T C}\right)$ images in IEC body phantom containing 6 fillable spheres. (A) PET and SPECT figures alone. (C) Same images fused with corresponding CT. T:B indicates actual target-to-background ratio for each experiment between concentration of radioactivity in spheres and in general compartment of phantom. (B) Quantitative results. Mean SUV within red region of interest $(\mathrm{ROI})$ in general background should be 1.0. Yellow ROI indicates SUV So $_{50}$ forgest (37-mm-diameter) sphere in both cases. Standard deviation quoted refers to variability about mean within ROI. Results are for single slice through phantom corresponding to central section through largest sphere. In this example, PET SUV 50 is slightly overestimated (7.9 [measured] vs. 7.3 [actual]) by about $8 \%$ whereas SPECT images are underestimated by about $11 \%$ (7.3 [measured] vs. 8.2 [actual]), probably because of partial-volume-effect losses caused by poorer spatial resolution in SPECT than in PET. Table 1 presents results for all spheres.

in total was approximately $300 \mathrm{MBq}$ for SPECT and 100 $\mathrm{MBq}$ for PET. These values, plus the volume of water in the phantom (stored as the patient weight), were entered into the acquisition protocol along with other pertinent information required, for use later in display and analysis. SUV was calculated as the mean of spheric volumes of interest defined by the CT scan for each sphere, to which was applied a threshold of $50 \%$ of the maximum value within the volume of interest $\left(\mathrm{SUV}_{50}\right)$. The results are shown in Table 1.

Although the SUV measured in a large background ROI in both cases was within $5 \%$ of the expected value of 1.0 , both systems suffered from underestimation of $\mathrm{SUV}_{50}$ in some (PET) or all (SPECT) spheres because of the finite spatial resolution of the systems. As noted previously, the SPECT spatial resolution is poorer than PET and thus would be expected to suffer larger underestimation when being used quantitatively in objects less than approximately 3 times the system spatial resolution. This is seen even for the largest (37 $\mathrm{mm}$ in diameter) sphere in SPECT, whereas partial-volume effect losses do not become significant ( $>5 \%$ underestimated) in the PET data until the sphere diameters are less than $17 \mathrm{~mm}$. The spatial resolution for this PET system is approximately $4.6 \mathrm{~mm}$ isotropic near the central axis. The SPECT reconstructed spatial resolution, on the other hand, is approximately $15 \mathrm{~mm}$ in full width at half maximum.

\section{Attenuation and Scatter Correction}

It is not the intention of this review to detail the various approaches to scatter correction or attenuation correction in SPECT, as these have largely been discussed previously (911); however, the incorporation of both is a necessity for quantitative SPECT reconstruction. The use of CT data for the purpose of attenuation correction in SPECT was reported nearly $30 \mathrm{y}$ ago by Moore (12) and predated the introduction of radionuclide transmission scanning schemes. Attenuation correction can readily be performed after reconstruction in SPECT using the algorithm of Chang (13) modified to use the measured body density (attenuation map) from the CT data, or the correction can be directly included in the iterative reconstruction algorithm. The scatter correction method that we have the most experience with, transmission-dependent scatter correction (14), has been implemented by several groups and found to give accurate results in a range of imaging situations (15-19). The simpler energy-based method known as the triple-energy-window approach (20) has also demonstrated accurate quantification (21) and is now offered by several $\gamma$-camera vendors. More sophisticated approaches that model the scatter have also been shown to give excellent results (22-25); however, they often rely on more intensive computing resources and specialized software.

Today, the intimate temporal and spatial integration of CT and emission tomographic devices has enhanced diagnostic accuracy and patient throughput (26-29). To these documented benefits we are now ready to add the ability to perform quantitatively accurate image reconstruction in SPECT using the CT data as the basis for the attenuation and scatter corrections.

Interestingly, the range of radionuclides investigated quantitatively with SPECT is already quite broad, including ${ }^{99 \mathrm{~m}} \mathrm{Tc},{ }^{111} \mathrm{In},{ }^{123} \mathrm{I},{ }^{131} \mathrm{I},{ }^{177} \mathrm{Lu},{ }^{186} \mathrm{Re}$, and ${ }^{201} \mathrm{Tl}$.

\section{Results Demonstrating Quantitative Accuracy in SPECT}

Although physicists often use phantoms in controlled experiments to demonstrate and refine the accuracy of image formation, we recognize that the situation when imaging the living human is very different and, hence, the excellent results often obtained in phantom experiments may not be as easy to replicate in humans. Therefore, in this paper we will predominantly discuss published validation work on SPECT studies in humans. For a summary of the accuracy of phantom experimental validation of quantitative SPECT, the reader is referred to Table 1 of the paper by Shcherbinin et al. (30) and Table 2 in the recently published MIRD pamphlet no. 23 on SPECT-based internal radionu- 
TABLE 1

SUVs for Compartments of IEC Phantom for SPECT and PET

\begin{tabular}{lcccc}
\hline Sphere diameter $(\mathrm{mm})$ & SPECT SUV (8.2) & Difference & PET SUV (7.3) & Difference \\
\hline Background (SUV $=1)$ & 0.96 & $-4.0 \%$ & 1.02 & +2.9 \\
37 & 7.3 & $-11.0 \%$ & 7.7 & $+7.6 \%$ \\
28 & 6.5 & $-21.3 \%$ & 7.6 & $+5.3 \%$ \\
22 & 4.5 & $-44.8 \%$ & 7.1 & $-3.5 \%$ \\
17 & 3.2 & $-60.6 \%$ & 6.2 & $-14.9 \%$ \\
13 & 2.9 & $-64.6 \%$ & 4.1 & $-43.7 \%$ \\
10 & 2.9 & $-65.2 \%$ & &
\end{tabular}

Impact on recovered SUV is more significant in SPECT than in PET, as SPECT systems have poorer spatial resolution. Both systems significantly underestimate SUV for sphere diameters of less than approximately 3 times the respective system spatial resolution.

clide dosimetry (31). Errors of up to approximately $20 \%$ in the estimation of the radioactivity concentration for a range of radionuclides are reported in these papers. As SPECT/ CT is the most convenient method for acquiring data suitable for producing quantitative SPECT images today, we will predominantly focus on reports using this multimodality device. In vivo validation of the quantitative accuracy of reconstructed images is challenging in the living human, but there are a limited number of imaging scenarios in which this can be achieved. Several of these are discussed below.

${ }^{99 m} T c$. Our group has published 2 papers using ${ }^{99 \mathrm{~m}} \mathrm{Tc}$ and SPECT/CT with in vivo validation in the clinical setting. The first demonstrated the accuracy of image reconstruction using ${ }^{99} \mathrm{~m}$ Tc-macroaggregated albumin in a lung perfusion scan, where it is assumed that close to $100 \%$ of the radiopharmaceutical is trapped in the lungs after intravenous injection of a calibrated amount of the radiopharmaceutical (8). This study allowed us to examine the accuracy of the total radioactivity within the images, but not the radioactivity concentration, and found that the total radioactivity in 12 subjects was estimated to be, on average, in error by $-1 \%$ (range, $-7 \%$ to $+4 \%$ ). The second clinical validation was in subjects undergoing left ventricular ejection fraction measurements with ${ }^{99 \mathrm{~m}}$ Tc-labeled erythrocytes. The radioconcentration of ${ }^{99 \mathrm{~m}} \mathrm{Tc}$ in a peripheral venous whole-blood sample at the time of the SPECT scan was measured in a $\gamma$-counter and compared with the concentration of the radiolabeled blood pool in the images reconstructed with CTbased attenuation and scatter correction (32). The accuracy was similar to the previous report, with the average error in estimated radioconcentration being $+1.3 \%$ and the range being $-6.3 \%$ to $+4.9 \%$. Our scatter correction approach (transmission-dependent scatter correction) uses software that has been developed in house. In yet another in vivo setting, Zeintl et al. have published the accuracy of quantitative ${ }^{99 \mathrm{~m}}$ Tc SPECT measuring the radioconcentration of urine in the bladder of subjects undergoing ${ }^{99 \mathrm{~m}} \mathrm{Tc}$-methylene diphosphate bone scans (33). Their reported average error was $+1.1 \%$, similar to what we have reported. Their approach used commercially available reconstruction software with attenuation and scatter correction.
${ }^{123} \mathrm{I}$. Kim et al. have examined the accuracy of quantitative ${ }^{123}$ I SPECT using transmission-dependent scatter correction and attenuation correction in the setting of brain imaging, initially in phantoms $(16,17)$. After validating the approach, the same group applied these techniques with ${ }^{123}$ I-iodoamphetamine for the measurement of regional cerebral blood flow in a multi-institutional setting (34). They measured the accuracy in phantoms and validated the SPECT regional cerebral blood flow results against those obtained with ${ }^{15} \mathrm{O}-\mathrm{H}_{2} \mathrm{O}$ PET. Across a range of different SPECT cameras, without coacquired CT scans for attenuation or scatter correction, they found a variation of about $\pm 5 \%$ in quantitative accuracy in phantoms. However, there was an overall average underestimation of $-12 \%$, which they attributed to septal penetration by higher-energy photons and the inability of their attenuation and scatter correction algorithms to estimate photon absorption due to the head holder or scanning couch. They found a slight underestimation in regional cerebral blood flow by iodoamphetamine SPECT, compared with ${ }^{15} \mathrm{O}-\mathrm{H}_{2} \mathrm{O}$ PET $(-6.1$ $\left.\mathrm{mL} \cdot 100 \mathrm{~g}^{-1} \cdot \mathrm{min}^{-1} ; r=0.88\right)$. Impressively, their quantitative SPECT methodology has been applied in more than 25,000 patients in more than 130 centers in Japan to date (34).

${ }^{201} \mathrm{Tl}$. We have investigated the accuracy achieved with ${ }^{201} \mathrm{Tl}$ for the imaging of brain malignancy. Although not able to validate the results in vivo in this situation, testing in an anthropomorphic striatal brain phantom (Radiology Support Devices) using the same methodology as for ${ }^{99 \mathrm{~m}} \mathrm{Tc}$ but with adjusted parameters for ${ }^{201} \mathrm{Tl}$ gave results within $\pm 4 \%$ of the true values for several different radioconcentrations. This method was subsequently applied to human subjects with suspected recurrent brain malignancies, and a correlation was found between lower SUV and prolonged survival (35). Iida et al. have also used quantitative ${ }^{201} \mathrm{Tl}$ SPECT in dynamic myocardial perfusion imaging to extract the uptake and washout kinetics of the tracer in dogs and compared their results against those for ${ }^{51}$ Co-microspheres (36). This was one of the first reports of fully quantitative kinetic analysis in SPECT of the myocardium and underlines the potential of new applications for quantitative SPECT.

Other Radionuclides. Several other radionuclides have been used quantitatively in SPECT, although in vivo validation 
has not been possible. These include ${ }^{111}$ In-labeled antibodies and tumor-seeking radiopharmaceuticals (37-39), ${ }^{131}$ I uptake in tumors $(40,41),{ }^{177} \mathrm{Lu}$ for dosimetry calculations in therapeutic applications $(42,43),{ }^{186} \mathrm{Re}$ in palliative bone cancer therapy (44), and even quantitative bremsstrahlung imaging of ${ }^{90} \mathrm{Y}(45)$.

\section{CLINICAL APPLICATIONS OF QUANTITATIVE SPECT}

A list of potential uses for quantitative SPECT is presented in Table 2. Few clinical applications of quantitative SPECT exist today because SPECT has generally been developed without routine application of corrections for attenuated and scattered radiation. The major application of attenuation correction in SPECT to date has been in the area of removing attenuation artifacts in SPECT myocardial perfusion imaging $(46,47)$, but the emphasis has not been on quantitative assessment. Likewise, in our institution we routinely apply attenuation correction to all clinical brain, abdominal, and pelvic soft-tissue SPECT (e.g., ${ }^{67} \mathrm{Ga}$ in infection or $\left[{ }^{123} \mathrm{I}\right.$ or $\left.\left.{ }^{131} \mathrm{I}\right]-\mathrm{MIBG}\right)$, but again this step is taken to mitigate attenuation artifacts or improve image quality rather than to pursue quantitative reconstruction per se.

\section{CHALLENGES FOR IMPLEMENTING QUANTITATIVE SPECT}

The evidence included in this review demonstrates that quantitative SPECT is potentially realizable using ${ }^{99 \mathrm{~m}} \mathrm{Tc}$, especially with today's current multimodality SPECT/CT scanners. The necessary hardware and software already ex- ist to implement quantitative SPECT and achieve reliable results likely to be, in general, within $\pm 10 \%$ of the true value in vivo over a wide variety of imaging conditions. The next step toward more widespread implementation is to introduce routine methodology in the SPECT systems to allow them to be calibrated for the specific radionuclide. In PET, all manufacturers provide procedures that allow the calibration factors for quantitative PET to be incorporated into the reconstruction software-calibration factors that are based on measurements of radioactivity using the site's own local dose calibrator. It is our experience in PET/CT that accuracy testing in a phantom can readily achieve values within $\pm 5 \%$ of the true value for the radioactivity concentration of ${ }^{18} \mathrm{~F}$ and ${ }^{68} \mathrm{Ga}\left(\mathrm{kBq} \cdot \mathrm{cm}^{-3}\right)$. We believe that the equivalent is needed for SPECT-that is, manufacturer-supplied techniques for calibration of the systems, defined operational limits within which quantitative SPECT can be applied, and routine validation of the technique as part of the regular SPECT quality control program.

Several considerations need to be addressed in the calibration process: first, measurements of system sensitivity, which will vary depending on the radionuclide, thickness of the scintillation crystal, collimator, and pulse height analyzer energy windows used; second, definition of any parameters required for the scatter correction algorithm, which will vary depending on the radionuclide and collimator; third, correction of deadtime losses; fourth, calibration of the pixel dimensions to allow scaling from pixels to unit volume $\left(\mathrm{cm}^{3}\right)$; and fifth, radionuclide half-life.

TABLE 2

List of Potential Uses for Quantitative SPECT

\begin{tabular}{|c|c|}
\hline Radiopharmaceutical/imaging test & Application \\
\hline $\begin{array}{l}\text { Planning/treatment (e.g., }{ }^{111} \mathrm{In},{ }^{123} \mathrm{I} \\
\left.\text { and }{ }^{177} \mathrm{Lu}\right)\end{array}$ & $\begin{array}{l}\text { Theranostics using quantitative SPECT for targeting and dosimetry planning in } \\
\text { monoclonal antibody, peptide, receptor, or other radionuclide therapy (e.g., } \\
\text { tositumomab, ibritumomab tiuxetan, and }{ }^{177} \mathrm{Lu} \text {-octreotate ) }(31,50,51)\end{array}$ \\
\hline $\begin{array}{l}\text { 99mTc myocardial perfusion } \\
\text { imaging }\end{array}$ & $\begin{array}{l}\text { Regional myocardial blood flow in } \mathrm{mL} \cdot 100 \mathrm{~g}^{-1} \cdot \mathrm{min}^{-1}(52) \text { or myocardial SUV for } \\
\text { detection of left main artery or balanced triple-vessel disease; measurement } \\
\text { of coronary flow reserve }\end{array}$ \\
\hline $\begin{array}{l}\text { 99m Tc functional lung scanning } \\
\text { (ventilation or perfusion) }\end{array}$ & Preoperative assessment of lobar function before surgical resection (53) \\
\hline 99mTc-macroaggregated albumin & $\begin{array}{l}\text { Treatment planning and measuring lung uptake }(54) \text { plus estimation radiation } \\
\text { dosimetry }(55) \text { in selective internal radionuclide therapy for liver cancer before } \\
\text { radioembolization }\end{array}$ \\
\hline 99mTc bone scanning & Quantitative measurement of uptake in metabolic bone disease (56) \\
\hline $\begin{array}{l}123 \text {-iodoamphetamine for regional } \\
\text { cerebral blood flow }\end{array}$ & Baseline/acetazolomide challenge in regional cerebral blood flow (34) \\
\hline 123|-labeled neuroreceptor tracers & Neurotransmitter brain imaging of receptor density and occupancy $(57,58)$ \\
\hline 123I-iodide thyroid uptake & $\begin{array}{l}\text { Determination of individualized doses for subsequent radioiodine }\left({ }^{131} \mathrm{I}\right) \text { therapy } \\
\text { and monitoring uptake in follow-up }\end{array}$ \\
\hline${ }^{131} \mathrm{I}$ in thyroid cancer & SUV in metastatic thyroid cancer and monitoring response to treatment \\
\hline $\begin{array}{l}{ }^{201} \text { TI myocardial perfusion } \\
\text { imaging }\end{array}$ & $\begin{array}{l}\text { Dynamic analysis of myocardial perfusion to derive kinetic parameters } K_{1} \text { and } \\
\text { distribution volume (36) }\end{array}$ \\
\hline General imaging & $\begin{array}{l}\text { Use of quantitative SPECT to monitor serial scans to assess disease } \\
\text { progression or response to treatment (e.g., }{ }^{67} \mathrm{Ga} \text { in infection) }\end{array}$ \\
\hline $\begin{array}{l}\text { Measuring radionuclide } \\
\text { biodistribution and dosimetry }\end{array}$ & $\begin{array}{l}\text { More accurate assessment of 3-dimensional biodistribution and radiation } \\
\text { dosimetry from new radiopharmaceuticals (31) }\end{array}$ \\
\hline
\end{tabular}




\section{QUALITY CONTROL FOR SPECT/CT}

Quality control for future quantitative SPECT will mirror that required by PET systems today, namely, a regular check of agreement between the dose calibrator and reconstructed SPECT values for radioactivity. Cross-calibration is slightly simpler for PET systems than for SPECT, because in PET all the radiation has the same photon energy and characteristics (namely $2 \times 511-\mathrm{keV}$ annihilation photons), independent of the radiotracer, whereas in SPECT different radiotracers-with different photon energies necessitating different collimators, pulse height analyzer energy windows, and other factors-will require separate calibrations and checking. With the cooperation of and implementation by the manufacturers of the SPECT/CT systems, many of the required parameters (such as for scatter correction) can be predefined and fixed for a particular radionuclide/collimator/pulse height analyzer setting. We envisage that strict adherence to the predetermined operating conditions and regular validation will be essential when quantitative SPECT is deployed. At least one example of such a protocol for a multicenter quantitative SPECT trial has been reported $(48,49)$.

\section{CONCLUSION}

Although SPECT is an extremely helpful, widely used clinical imaging modality, it will not achieve its full potential when it is used in a purely qualitative manner. We believe that combined SPECT/CT is a game-changer in numerous ways and provides the impetus for a paradigm shift in SPECT use into the quantitative domain in radionuclide emission tomography - a position occupied exclusively by PET until now. As we have demonstrated in this paper, users should also be aware of the deterioration in quantitative accuracy with decreasing object size when objects below 3 times the spatial resolution of the system are imaged. Nevertheless, cooperation between researchers developing the methodology required for quantitative SPECT and manufacturers will allow its wider introduction for clinical use.

In summary, the time for the clinical implementation of quantitative SPECT has come.

\section{ACKNOWLEDGMENTS}

We are grateful to Professor Hidehiro Iida of the National Cardiovascular Centre Research Institute in Osaka, Japan, for useful discussions and for providing an update on the Japanese brain imaging studies, and we thank our local colleagues for useful suggestions of potential clinical applications for quantitative SPECT.

\section{REFERENCES}

1. Pfeifer A, Knigge U, Mortensen J, et al. Clinical PET of neuroendocrine tumors using ${ }^{64} \mathrm{Cu}$-DOTATATE: first-in-humans study. J Nucl Med. 2012;53:1207-1215.

2. Bailey DL, Hutton BF, Walker PJ. Improved SPECT using simultaneous emission and transmission tomography. J Nucl Med. 1987;28:844-851.
3. Tung C-H, Gullberg GT, Zeng GL, et al. Non-uniform attenuation correction using simultaneous transmission and emission converging tomography. IEEE Trans Nucl Sci. 1992;39:1134-1143.

4. Hudson HM, Larkin RS. Accelerated image reconstruction using ordered subsets of projection data. IEEE Trans Med Imaging. 1994;13:601-609.

5. Bailey DL, Roach PJ, Bailey EA, et al. Development of a cost-effective modular SPECT/CT scanner. Eur J Nucl Med Mol Imaging. 2007;34:1415-1426.

6. Jakoby BW, Bercier Y, Conti M, et al. Physical and clinical performance of the mCT time-of-flight PET/CT scanner. Phys Med Biol. 2011;56:23752389

7. NEMA. Performance Measurements of Positron Emission Tomographs. Washington, DC: National Electrical Manufacturers Association; 2007. Report no. NU2-2007.

8. Willowson K, Bailey DL, Baldock C. Quantitative SPECT using CT-derived corrections. Phys Med Biol. 2008;53:3099-3112.

9. Bailey DL. Transmission scanning in emission tomography. Eur J Nucl Med. 1998;25:774-787.

10. King MA, Glick SJ, Pretorius PH, et al. Attenuation, scatter, and spatial resolution compensation in SPECT. In: Wernick MN, Aarsvold JN, eds. Emission Tomography: The Fundamentals of PET and SPECT. Waltham, MA: Elsevier Academic Press; 2004:473-498.

11. Vandervoort E, Celler A, Harrop R. Implementation of an iterative scatter correction, the influence of attenuation map quality and their effect on absolute quantitation in SPECT. Phys Med Biol. 2007;52:1527-1545.

12. Moore SC. Attenuation compensation. In: Ell PJ, Holman BL, eds. Computed Emission Tomography. London, U.K.: Oxford University Press; 1982:339-360.

13. Chang LT. A method for attenuation correction in radionuclide computed tomography. IEEE Trans Nucl Sci. 1978;NS-25:638-643.

14. Meikle SR, Hutton BF, Bailey DL. A transmission dependent method for scatter correction in SPECT. J Nucl Med. 1994;35:360-367.

15. Iida H, Narita $Y$, Kado H, et al. Effects of scatter and attenuation correction on quantitative assessment of regional cerebral blood flow with SPECT. J Nucl Med. 1998;39:181-189.

16. Kim KM, Watabe H, Shidahara M, et al. SPECT collimator dependency of scatter and validation of transmission-dependent scatter compensation methodologies. IEEE Trans Nucl Sci. 2001;NS-48:689-696.

17. Kim KM, Varrone A, Watabe $\mathrm{H}$, et al. Contribution of scatter and attenuation compensation to SPECT images of nonuniformly distributed brain activities. $J$ Nucl Med. 2003;44:512-519.

18. Larsson A, Johansson L. Scatter-to-primary based scatter fractions for transmission-dependent convolution subtraction of SPECT images. Phys Med Biol. 2003;48:N328-N328.

19. Narita Y, Eberl S, Iida H, et al. Monte Carlo and experimental evaluation of accuracy and noise properties of two scatter correction methods for SPECT. Phys Med Biol. 1996;41:2481-2496.

20. Ogawa K, Harata Y, Ichihara T, et al. A practical method for position-dependent Compton-scatter correction in single photon emission CT. IEEE Trans Med Imaging. 1991;10:408-412.

21. Ichihara T, Ogawa K, Motomura N, et al. Compton scatter compensation using the triple-energy window method for single- and dual-isotope SPECT. $J$ Nucl Med. 1993;34:2216-2221.

22. Beekman FJ, Kamphuis C, Frey EC. Scatter compensation methods in 3D iterative SPECT reconstruction: a simulation study. Phys Med Biol. 1997;42:16191632.

23. Kadrmas DJ, Frey EC, Karimi SS, et al. Fast implementations of reconstructionbased scatter compensation in fully 3D SPECT image reconstruction. Phys Med Biol. 1998;43:857-873.

24. Buvat I, Rodrigues-Villafuerte M, Todd-Pokropek A, et al. Comparative assessment of nine scatter correction methods based on spectral analysis using Monte Carlo simulations. J Nucl Med. 1995;36:1476-1488.

25. El Fakhri G, Buvat I, Benali H, et al. Relative impact of scatter, collimator response, attenuation, and finite spatial resolution corrections in cardiac SPECT. J Nucl Med. 2000;41:1400-1408.

26. Schillaci O, Simonetti G. Fusion imaging in nuclear medicine: applications of dual-modality systems in oncology. Cancer Biother Radiopharm. 2004;19:1-10.

27. Schillaci O. Hybrid SPECT/CT: a new era for SPECT imaging? Eur J Nucl Med Mol Imaging. 2005;32:521-524.

28. Roach PJ, Schembri GP, Ho Shon IA, et al. SPECT/CT imaging using a spiral CT scanner for anatomical localization: impact on diagnostic accuracy and reporter confidence in clinical practice. Nucl Med Commun. 2006;27:977-987.

29. Mariani G, Bruselli L, Kuwert T, et al. A review on the clinical uses of SPECT/ CT. Eur J Nucl Med Mol Imaging. 2010;37:1959-1985.

30. Shcherbinin S, Celler A, Belhocine T, et al. Accuracy of quantitative reconstructions in SPECT/CT imaging. Phys Med Biol. 2008;53:4595-4604. 
31. Dewaraja YK, Frey EC, Sgouros G, et al. MIRD pamphlet no. 23: quantitative SPECT for patient-specific 3-dimensional dosimetry in internal radionuclide therapy. J Nucl Med. 2012;53:1310-1325.

32. Willowson K, Bailey DL, Baldock C, et al. In vivo validation of quantitative SPECT in the heart. Clin Physiol Funct Imaging. 2010;30:214-219.

33. Zeintl J, Vija AH, Yahil A, et al. Quantitative accuracy of clinical ${ }^{99 \mathrm{~m} T c}$ SPECT/ CT using ordered-subset expectation maximization with 3-dimensional resolution recovery, attenuation, and scatter correction. J Nucl Med. 2010;51:921-928.

34. Iida H, Nakagawara J, Hayashida K, et al. Multicenter evaluation of a standardized protocol for rest and acetazolamide cerebral blood flow assessment using a quantitative SPECT reconstruction program and split-dose ${ }^{123}$ I-iodoamphetamine. J Nucl Med. 2010;51:1624-1631.

35. Willowson K, Bailey DL, Schembri G, et al. CT-based quantitative SPECT for the radionuclide ${ }^{201} \mathrm{Tl}$ : experimental validation and a standardized uptake value for brain tumour patients. Cancer Imaging. 2012;12:31-40.

36. Iida H, Eberl S, Kim KM, et al. Absolute quantitation of myocardial blood flow with ${ }^{201} \mathrm{Tl}$ and dynamic SPECT in canine: optimisation and validation of kinetic modelling. Eur J Nucl Med Mol Imaging. 2008;35:896-905.

37. He B, Du Y, Song X, et al. A Monte Carlo and physical phantom evaluation of quantitative In-111 SPECT. Phys Med Biol. 2005;50:4169-4185.

38. He B, Frey EC. Comparison of conventional, model-based quantitative planar, and quantitative SPECT image processing methods for organ activity estimation using In-111 agents. Phys Med Biol. 2006;51:3967-3981.

39. Ljungberg M, Frey E, Sjogreen K, et al. 3D absorbed dose calculations based on SPECT: evaluation for 111-In/90-Y therapy using Monte Carlo simulations. Cancer Biother Radiopharm. 2003;18:99-107.

40. Israel O, Iosilevsky G, Front D, et al. SPECT quantitation of iodine-131 concentration in phantoms and human tumors. J Nucl Med. 1990;31:1945-1949.

41. Dewaraja YK, Wilderman SJ, Ljungberg M, et al. Accurate dosimetry in ${ }^{131} \mathrm{I}$ radionuclide therapy using patient-specific, 3-dimensional methods for SPECT reconstruction and absorbed dose calculation. J Nucl Med. 2005;46:840-849.

42. Beauregard JM, Hofman MS, Pereira JM, et al. Quantitative ${ }^{177}$ Lu SPECT (QSPECT) imaging using a commercially available SPECT/CT system. Cancer Imaging. 2011;11:56-66.

43. Pereira JM, Stabin MG, Lima FR, et al. Image quantification for radiation dose calculations: limitations and uncertainties. Health Phys. 2010;99:688-701.

44. Israel O, Keidar Z, Rubinov R, et al. Quantitative bone single-photon emission computed tomography for prediction of pain relief in metastatic bone disease treated with rhenium-186 etidronate. J Clin Oncol. 2000;18:2747-2754.
45. Rong X, Du Y, Ljungberg M, et al. Development and evaluation of an improved quantitative ${ }^{90} \mathrm{Y}$ bremsstrahlung SPECT method. Med Phys. 2012;39:2346-2358.

46. Heller GV, Bateman T, Johnson L, et al. Clinical value of attenuation correction in stress-only Tc-99m sestamibi SPECT imaging. J Nucl Cardiol. 2004;11:273-281.

47. Heller GV, Links J, Bateman T, et al. American Society of Nuclear Cardiology and Society of Nuclear Medicine joint position statement: attenuation correction of myocardial perfusion SPECT scintigraphy. J Nucl Cardiol. 2004;11:229-230.

48. Tossici-Bolt L, Dickson JC, Sera T, et al. Calibration of gamma camera systems for a multicentre European ${ }^{123}$ I-FP-CIT SPECT normal database. Eur J Nucl Med Mol Imaging. 2011;38:1529-1540.

49. Dickson JC, Tossici-Bolt L, Sera T, et al. Proposal for the standardisation of multi-centre trials in nuclear medicine imaging: prerequisites for a European ${ }^{123}$ I-FP-CIT SPECT database. Eur J Nucl Med Mol Imaging. 2012;39:188-197.

50. Bodei L, Ferone D, Grana CM, et al. Peptide receptor therapies in neuroendocrine tumors. J Endocrinol Invest. 2009;32:360-369.

51. Ljungberg M, Sjogreen K, Liu X, et al. A 3-dimensional absorbed dose calculation method based on quantitative SPECT for radionuclide therapy: evaluation for ${ }^{131}$ I using Monte Carlo simulation. J Nucl Med. 2002;43:1101-1109.

52. Da Silva AJ, Tang HR, Wong KH, et al. Absolute quantification of regional myocardial uptake of ${ }^{99 \mathrm{~m}} \mathrm{Tc}$-sestamibi with SPECT: experimental validation in a porcine model. J Nucl Med. 2001;42:772-779.

53. Ohno Y, Koyama H, Nogami M, et al. Postoperative lung function in lung cancer patients: comparative analysis of predictive capability of MRI, CT, and SPECT. AJR. 2007; 189:400-408.

54. Willowson K, Bailey DL, Baldock C. Quantifying lung shunting during planning for radio-embolization. Phys Med Biol. 2011;56:N145-N152.

55. Garin E, Lenoir L, Rolland Y, et al. Dosimetry based on ${ }^{99 \mathrm{~m}} \mathrm{Tc}-$ macroaggregated albumin SPECT/CT accurately predicts tumor response and survival in hepatocellular carcinoma patients treated with ${ }^{90} \mathrm{Y}$-loaded glass microspheres: preliminary results. $J$ Nucl Med. 2012;53:255-263.

56. Israel $\mathrm{O}$, Front $\mathrm{D}$, Hardoff $\mathrm{R}$, et al. In vivo SPECT quantitation of bone metabolism in hyperparathyroidism and thyrotoxicosis. J Nucl Med. 1991;32:11571161.

57. Fujita M, Ichise M, van Dyck CH, et al. Quantification of nicotinic acetylcholine receptors in human brain using [ ${ }^{123}$ I] 5-I-A-85380 SPET. Eur J Nucl Med Mol Imaging. 2003;30:1620-1629.

58. Fujita M, Varrone A, Kim KM, et al. Effect of scatter correction on the compartmental measurement of striatal and extrastriatal dopamine D2 receptors using [ ${ }^{123}$ I] epidepride SPET. Eur J Nucl Med Mol Imaging. 2004;31:644-654. 